With the accuracy hitherto obtained it is, however, impossible to decide with certainty if this difference really must be ascribed to a $2 p$ and a $3 p$ orbit (the only other energy difference of the potassium spectrum which might come into consideration as being of about the same magnitude is $I s-2 p$ corresponding to $\mathrm{I} \cdot 6$ volts). Other eventual edges corresponding to higher $p$-orbits lie too close together to be separated even with calcite.

Experiments are being started with the X-ray absorption spectrum of neon, for which fine structures are expected corresponding to energy differences in the optical spectrum of sodium. As in this case the separation is expected to be much larger than in the case of argon, we hope to be able to decide some questions which still remained unsettled in our experiments with argon.

D. Coster.

J. H. VAN DER TUUK.

Physical Laboratory, University, Groningen.

\section{Spinning Electrons and the Structure of Spectra.}

GoudsmiT and Uhlenbeck have suggested that the structure of multiple lines in spectra can be made much more comprehensible if it is assumed that the electron itself has a magnetic moment of one Bohr magneton $(\mathrm{eh} / 4 \pi \mathrm{mc})$ and an angular momentum of one-half unit $(h / 4 \pi)$, which can orient itself in various ways with respect to the normal to the electron orbits. Goudsmit and Uhlenbeck point out (Naturwissenschaften, Nov. 20, I925) that to obtain such a magnetic moment from the rotation of a Lorentz electron about its axis, there would have to be a peripheral velocity greatly exceeding the speed of light. It can also be easily seen that the magnetic energy with its resulting mass would be much greater than the electrical energy. These difficulties do not seem to be easy to overcome by any small modification of the classical model of the electron. It appears, however, that an entirely different picture of electronic structure, based on the idea of light quanta, is capable of giving a consistent explanation of the energy of the electron, and also of predicting the values of some of the electronic constants.

The author has suggested that the energy in light waves consists of corpuscular quanta which are guided by a virtual field, along Poynting's vector or some similar direction. In general, the direction could not be just that of Poynting's vector, for that does not transform as a velocity under the Lorentz transformation. In the particular case where $E$ and $H$ are equal and at right angles, however, as for a wave in free space, a vector of magnitude $c$ along Poynting's vector may be proved to transform as a velocity, so that for this case it can be taken as the velocity vector of the quanta. A defect in such a scheme was the existence of the constitutive energy of the electrons, which presumably is energy in the field, although otherwise the field was not supposed to carry energy. The present suggestion is that the electron's field itself is virtual, and that its energy consists of a quantum, which is permanently attached to it, revolving about it. To make this consistent with the radiation theory, the path of the electronic quantum should be determined in the same way as the path of a quantum in a radiation field. This can be done; for if we imagine a virtual field, consisting of an electrical point singularity $e$, and a magnetic doublet singularity $\mathrm{ch} / 4 \pi m c$, there is a circle in the equatorial plane at every point of which Poynting's vector is tangential to the circle and satisfies the particular conditions given above, so that this orbit can be the path of a quantum. The circle has a radius $r_{0}$ satisfying the condition that the electric field at this distance, $e / r_{0}{ }^{2}$, should equal in magnitude the magnetic field, $e h / 4 \pi m c r_{0}{ }^{3}$, which gives $r_{0}=$ $h / 4 \pi m c=\mathrm{I} \cdot 93 \times 10^{-11} \mathrm{~cm}$. Evidently at points of this circle the fields are perpendicular to each other, and Poynting's vector is tangential. The picture of the electron is then an energyless field from a point charge and doublet, with the energy and angular momentum located in the quantum travelling with the speed of light in a circle of radius $r_{0}$.

We assume the energy of the quantum to be given by the quantum condition for a rotator in its first state, $E=h \nu / 2$, where $\nu$ is the frequency of rotation. Since the quantum travels with the velocity of light, this frequency is $c / 2 \pi r_{0}$. Substituting for $r_{0}$, this is $\nu=2 m c^{2} / h$, giving from the quantum condition $E=h \nu / 2=m c^{2}$. The mass of this energy is $E / c^{2}=m$, so that the quantum carries just the mass of the electron. The angular momentum is equal to $m r_{0} c$; again substituting for $r_{0}$, this equals $h / 4 \pi$. Thus we obtain a rational explanation of the half-quantum value of the angular momentum, and of its anomalous ratio to the magnetic moment.

In attempting to work out the dynamics of the paths of quanta, many interesting questions present themselves. It is necessary to consider that there are forces acting on quanta which must operate when the quanta change their direction and hence their momentum. The reactions of these forces on atoms may in some cases be the "quantum forces" which the author has suggested for changing the atom from one stationary state to another in a quantum transition. It is hoped that a further study of these forces will provide more definite information about the dynamics of an electron such as has been here suggested.

Jefferson Physical Laboratory, Harvard University, March I8.

\section{Mercury Helides.}

In a first note (Nature, December I3, I924, p. 86I) $\mathrm{I}$ announced the synthesis of mercury helide. In a second note (NATURE, March 7, 1925) the empirical formula of the compound was given as $\mathrm{HgHe}$, and afterwards (NATURE, June 20, I925, p. 947) the formula $\mathrm{HgHe}_{10}$ was substituted for the earlier one. Details of the methods and apparatus used for synthesizing the compound were given in a paper communicated to the Royal Society last July. The chief points of interest with which that paper dealt may be conveniently enumerated as follows:

r. The formation of the helide is accompanied by a decrease in the pressure of the helium.

2. The maximum decrease in pressure during any synthesis was never greater than 3.3 per cent. of the initial pressure of $6 \mathrm{~mm}$.

3. At ordinary temperatures the helide possesses stability; but on passing the gas over a red-hot platinum spiral it is completely resolved into its components; and as a result of the decomposition the helium is restored to its original volume and pressure.

4. The compound is neither liquefied nor absorbed by charcoal at the temperature of liquid air.

5. In a quantitative analysis of the helide, the total weight of the mercury obtained was 0.00021079 gm.

6. Experiment proved that the helide is most readily formed when the pressure of the helium approximates $6 \mathrm{~mm}$. of mercury.

7. Qualitative experiments made with a Jamin 